\title{
The Frequency of Selected Polymorphic Variants of the RET Gene in Patients with Medullary Thyroid Carcinoma and in the General Population of Central Poland.
}

M. Sromek ${ }^{1}$ MSc, M. Czetwertyńska² MD, E. Skasko PhD, J. Zielińska³ MSc D. Czapczak ${ }^{1}$ MSc, J. Steffen Prof.

${ }^{1}$ Department of Endocrinology, Maria Sklodowska-Curie Memorial Cancer Center and Institute of Oncology, Warsaw, Poland

${ }^{2}$ Department of Nuclear Medicine and Endocrine Oncology, Maria Sklodowska-Curie Memorial Cancer Center and Institute of Oncology, Warsaw, Poland

${ }^{3}$ Bioinformatics Department, Institute of Biochemistry and Biophysics, Polish Academy of Sciences, Warsaw, Poland.

${ }^{4}$ Department of Immunology, Maria Sklodowska-Curie Memorial Cancer Center and Institute of Oncology, Warsaw, Poland

Short title (running head): Polymorphic variants of RET gene.

Keywords: single-nucleotide polymorphisms, sporadic medullary thyroid carcinoma, mutation of the RET gene, mRNA sequence.

Address for correspondence:

Maria Sromek, MSc, Endocrinology Department, Maria Sklodowska-Curie Memorial Cancer Center and Institute of Oncology, W.K. Roentgen 5, 02-781 Warsaw, Poland.

Fax: 64490 85; telephone: +48 2254626 90; e-mail: mariasromek@coi.waw.pl

Word counts; text: 3075

The number of figures and tables; figures:2, tables: 5 


\begin{abstract}
OBJECT: The object of this work was to compare the frequency of three polymorphic changes in the RET proto-oncogene: L769L and S836S and S904S in patients with medullary thyroid carcinoma (MTC) $(n=246)$ and in the general population $(n=420$ for SNP L769L and S904S; $n=411$ for SNP 836). We tried to investigate how the harboured SNPS affect the age at onset of SMTC and MTC in carriers of known pathogenic mutations at codons 634 and 791 of the RET gene. RESULTS: A statistically significant difference was found in the frequency of the heterozygous change L769L in patients with SMTC (48.3\%) and in unaffected individuals (39.5\%). CONCLUSIONS: The presence of the polymorphic change L769L in the RET gene predisposes to the development of sporadic medullary thyroid carcinoma and also lowers the age of onset of MTC in carriers of the homozygous polymorphic variant L769L. The presence of this polymorphic change in MTC patients carrying at the same time the RET codon 634 mutation lowers the age of onset of MTC in this group.
\end{abstract}

\title{
INTRODUCTION
}

Medullary thyroid carcinoma (MTC) is a malignant tumor originating from parafollicular C cells that produce calcitonin and accounts for $5-10 \%$ of all thyroid cancers. MTC develops in either sporadic $(75 \%)$ or hereditary form $(25 \%)$. In the inherited form, MTC appears in three cancer syndromes: FMTC (familial medullary thyroid carcinoma), MEN2A and MEN2B (multiple endocrine neoplasia type 2).

The hereditary form of MTC is associated with the development of germline changes in the RET gene. The RET (REarranged during Transfection) gene localized to 10q11.2 was identified in 1985 (1). The RET gene encodes a receptor tyrosine kinase and is expressed in the neural crest cells and neural crest-derived tissues $(2,3)$. The germline mutations of the RET gene causing increased receptor activation (gain-of-function mutations) are responsible for the FMTC, MEN2A (e.g. exon 10, codons 609, 611, 620) and MEN2B (e.g. exon 16, codon 918) cancer syndromes, while germline mutations of the RET gene leading to loss of 
receptor tyrosine kinase activity (loss-of-function mutations) are responsible for the development of Hirschsprung's disease $(4,5)$.

In our earlier article we presented a spectrum of the RET gene mutations prevalent in the population of Central Poland (6). It represents the majority of mutations of this gene reported in the world literature $(7,8,9)$. The highest number of mutations were identified at codons $634(26.8 \%)$ and $791(20.9 \%)$.

In the RET gene, apart from clearly pathogenic mutations, also polymorphic changes in the DNA sequence were found. These are frequent changes (frequency above 1\%) of several nucleotides in the DNA of the human population. They are divided into: mini and microsatellite sequences polymorphisms and single nucleotide polymorphisms (SNPs). SNPs make up about $90 \%$ of all the human genome variations and occur every 100 to 300 bases along the 3-billion-base human genome (10).

A SNP in which the amino acid sequence of protein that is produced is altered due to single nucleotide polymorphism is termed non-synonymous. Synonymous SNPs do not lead to a change in the amino acid sequence. Studies conducted over the past few years revealed the effect of synonymous mutations on predisposition to develop cancer. To date, several types of SNPs have been identified in the RET gene: G691S/exon 11, GTT/AGT; L769L/exon 13, CTT/CTG; S836S/exon 14, AGC/AGT; S904S/exon 15, TCC/TCG. These changes were present both in affected individuals (endocrine tumors, MTC, PTC, HSCR) as well as in the non-affected population $(11,12)$. Among the several known SNPs, only one change, in exon 11 G691S, results in the substitution of another amino acid in the protein chain (13).

In our group of patients three types of single-nucleotide polymorphisms were found in the RET gene in exons 13 (L769L), 14 (S836S) and 15 (S904S). These molecular variants appeared in one or both alleles of affected and non-affected individuals.

The aim of our study was to compare the frequency of occurrence of three SNPs: L769L, S836S and S904S, in patients with sporadic medullary thyroid carcinoma and in nonaffected individuals. Furthermore, we studied the effect of carrying homo- and heterozygous 
polymorphic variant $\mathrm{L} 769 \mathrm{~L}$ on the age at onset of the sporadic and familial medullary thyroid carcinoma.

\section{MATERIALS AND METHODS}

\section{$\underline{\text { Patients }}$}

246 patients, from Central Poland, with histological changes consistent with medullary thyroid cancer (MTC), treated at the Warsaw Oncology Centre in the years 1998-2007, were assessed. Out of that number, 217 were not found to be carriers of the known pathogenic mutations and were diagnosed as having sporadic medullary thyroid cancer. All patients with diagnosed SMTC were examined in respect of occurrence of other germline mutations in exons: $10,11,13,14,15$ and 16, in which changes connected with hereditary MTC were detected. The other 29 patients were found to be carriers of mutations in codons 791 (9 patients) and 634 (19 patients).

Assessments were also performed in 11 non-affected carriers of codon 791 mutations and 3 non-affected carriers of codon 634 mutations.

All patients were subjected to the following standard diagnostic procedures: laboratory tests for serum CEA and calcitonin (basal and in some cases also stimulated levels), calcium, PTH, catecholamines and their urinary metabolites, neck ultrasound scanning, abdominal ultrasound scanning including adrenal gland area evaluation, echocardiography and ABP (ambulatory blood pressure monitoring). Peripheral blood sample was taken for genetic testing.

The frequency of occurrence of molecular variants in the population was evaluated by testing anonymous blood samples of neonates from Central Poland. The presence of the L769L and S904S molecular variants were analyzed in 420 blood samples and of the S836S molecular variant in 411 blood samples.

Methods

The genomic DNA of the studied individuals was isolated from peripheral blood lymphocytes. Four fragments of the RET gene: exons 13, 14, 15 and 11 were amplified using 
the PCR technique, in which the frequency of occurrence of SNP was studied. The product of the sequencing reaction was subjected to electrophoresis on $5 \%$ denaturing polyacrylamide gel in a Perkin Elmer ABI Prism 377 DNA sequencer. Detailed information can be found in publication Paszko et al 2007 (6).

\section{Statistical Analysis}

The statistical analysis of the frequency of polymorphic variants was performed using Chi-square $\left(X^{2}\right)$ test with Yates' Correction. The difference calculated with the probability margin of $p<0.05$ was deemed statistically significant. When analyzing the age at onset of the disease in the studied groups, median ages at onset were compared using Mann-Whitney test.

\section{Bioinformatics Analysis}

mRNA sequence was taken from NCBI nucleic database, divided to shorter parts exons - and folded by RNAfold software from RNA ViennaPackage (14). RNAfolding algorithm counts energy parameters, and their values depend on sequence length. The reason is that if RNA molecule is long enough, the probability of the predicted structure with minimal free energy (MFE) is very low, because the amount of possible secondary structure grows expotentially with the length of the sequence. In these experiments we wanted to observe the possible influence of one nucleotide change on the energy of the sequence. This change cannot be observed if we consider the whole RET sequence - its length is 3345 nucleotides. Considering small fragments, allows us to observe local energy parameters and stabilization differences. The structures were visualized by VARNA software (Visualization Applet for RNA) (15).

\section{RESULTS}

A total of 124 cases of homo- and heterozygous molecular variants L769L (57.14\%) were identified in the 217 MTC patients assessed; 202 cases of homo- and heterozygous molecular variants L769L (48.09\%) were identified in the 420 samples collected from the control group, OR: 1.43; $p=0.0374$. The frequency of the individual alleles in the case of the 
polymorphic change $\mathrm{L} 769 \mathrm{~L}$ differed from the frequency of these alleles in the control group. X2 statistical analysis showed these differences to be statistically significant, OR: 1.43; $\mathrm{p}=0.0394$, Table 1 .

The analysis of the polymorphic variant S836S in exon 14 and S904S in exon 15 of the RET gene showed no statistically significant difference in the frequency of heterozygotes and homozygotes between MTC patients and non-affected individuals. Table 1.

Furthermore, we analyzed whether the presence of the polymorphic change $\mathrm{L} 769 \mathrm{~L}$ in patients with sporadic medullary thyroid carcinoma affected the age at onset of the disease. Thus, using Mann-Whitney test we compared the median ages at onset of the disease in the three groups of MTC patients carrying different polymorphic variants: CTT/CTT, CTT/CTG and CTG/CTG, Table 2. The results showed that the age at onset of the disease in carriers of the homozygous polymorphic change CTG/CTG was 11.5 years lower than the age at onset in individuals with the wild-type nucleotide sequence CTT/CTT. The difference between the median ages at onset between these two groups was statistically significant $(p=0.021)$. Similarly, the age at onset in carriers of the homozygous change CTG/CTG was 14.5 years lower than the age of affected carriers of heterozygous polymorphic change CTT/CTG. The difference between the compared groups was also statistically significant $(p=0.015)$, Table 2 .

In the studied group of MTC patients the polymorphic change L769L was accompanied by a F791Y mutation in exon 13. Each patient carrying mutation F791Y had at the same time SNP L769L, Table 3. The most frequently occurring mutation in the studied patients was the RET exon 11 codon 634 mutation. In carriers of these mutations at codon 634 (C634R, C634S, C634G) the polymorphic change L769L occurred in only 59,0\% of cases, Table 3. The age at onset in carriers of RET codon 634 mutations and SNP L769L was 23 years lower than in patients carrying only the mutation at codon 634 . The difference between the median ages at onset in both groups of patients was significant $p=0.0072$, Fig. 1

The obtained results induced us to carry out bioinformatics analysis of the changes that may occur during genetic information reading of the studied RET gene fragments. The 
simplest prediction of mRNA structure is a prediction of thermodynamic stable structure, minimal free energy structure (MFE). The result of this method is shown in Table 4. Differences in minimal free energy between wild types and mutants are less than $5 \%$ in the case of SNP's S904S, S836S and mutations Y791F and C634R. No effect on MFE is visible also in the combination of C634R and SNP L769L.

Only in the case of exon 13 and SNP the difference is noticeable. SNP reduces the energy of the wild-type by $17 \%$, and the mutant Y791F by $7 \%$. It can be concluded that only SNP $\mathrm{L} 769 \mathrm{~L}$ reduces the minimal free energy of small mRNA fragment (in the longer part of sequence - from exon 11 to 13 , the effect is not visible). Fig. 2 shows the predicted MFE structures of exon 13. The effect of one mutated nucleotide is visible - it changes the MFE structure in wild type and mutated sequence.

However, it is known that mRNA is unlikely to adopt a single stable conformation. It rather exists as a population of structures - RNA molecules fold to a metastate, which alternates between a number of closely related structures (16). Therefore recently ensemblebased approaches to structure prediction are more popular. The results of ensemble diversity and the free energy of the thermodynamic ensemble are shown also in Table 4. Evident differences between exons are visible in case of ensemble diversity (the average base-pair distance between all structures in the Boltzmann ensemble). The base-pair distance is defined as the number of base-pairs not shared by two structures. Lower ensemble diversity means that more base pairs are shared in the ensemble of predicted structures, so RNA structure is more stable.

All tested mutations reduced the value of ensemble diversity. Pathogenic mutations have negligible effect: Y791F reduce the value by $6.6 \%$ and C634R only by $1.86 \%$. On the other hand, SNPs reduced S836S by $13 \%$ and SNP S904S by $29 \%$.

Greater change is visible in the fragment of joined exons $(11,12,13)$ : SNP L769L reduces the ensemble diversity of exon 13 by $10.68 \%$ and of exon having a pathogenic mutation (C634R) by 10.91\%. Again, the greatest change is visible in exon 13: SNP L769L reduces 
the ensemble diversity of exon 13 up to $76 \%$ and of exon having a pathogenic mutation (Y791F) up to 53\%.

\section{DISCUSSION}

The assessment of the occurrence of the three polymorphic changes L769L, S836S and S904S in patients with sporadic medullary thyroid carcinoma and in non-affected individuals, demonstrated in our study differences in the frequency of polymorphism L769L in exon 13 of the RET gene. The differences in the frequency of heterozygous changes (CTT/CTG) were statistically significant as determined by the $\chi^{2}$ statistical analysis. Many authors differ in their opinions as to the effect of the polymorphic variants on the predisposition to develop sporadic MTC. Several authors found no significant differences in the frequency of polymorphic changes L769L, S836S and S904S in patients with sporadic and familial medullary thyroid carcinoma as compared with the control group $(17,18,19,20$, $21,22,23$, ). Baumgartner-Parzer et al. (12), suggested that the discrepancy in the results of studies on the occurrence of polymorphic variants in exons 13 and 14 of the RET gene were due to ethnic differences in the studied population of MTC patients or differences in the criteria of selection of the assessed MTC patients and in particular in the selection of individuals for the control group.

The frequency of the polymorphic change L769L in exon 13 in the MTC affected and non-affected population of Central Poland was found to be higher then the frequency of these changes in other countries, Table 1 . The highest frequency of the polymorphic change L769L has so far been observed in Turkey and it was $29 \%$ of the MTC-affected population. The least frequent occurrence of SNP L769L was reported in the population of Brazil where it accounted for $13 \%$ of MTC patients and in Portugal (16\% and $18 \%$ in MTC patient population and non-affected individuals, respectively) (20). Studies performed by Wiench et al. in the Polish population of Silesia Province in 2004 showed the frequency of polymorphism L769L in MTC patients and the control group to be $23 \%$ and $27 \%$, respectively. The dissimilarity of results obtained by Wiench et al. (22) and in our study was most likely due to regional differences in the frequency of the polymorphic variants in the Polish population. 
An important aspect of our study was to investigate the effect of the presence of the polymorphic change $\mathrm{L} 769 \mathrm{~L}$ in the RET gene on the age at onset in patients with sporadic MTC. We found that the age at onset in carriers of the homozygous change L769L was significantly lower than in carriers of the wild nucleotide, Table 2. These findings clearly showed that homozygous polymorphic changes could act as modifiers in the development of sporadic medullary thyroid carcinoma. Wiench et al. (22) found increased frequency of the L769L polymorphism in younger (aged <30) patients with sporadic MTC from Silesia Province, however, the difference in frequency compared with the control group was not statistically significant. Gursoy et al. (20) however, found no significant differences in the age at onset of sporadic MTC between younger patients (under 40 years of age) carrying polymorphism L769L and the group of older patients (over 40 years of age).

We have made an interesting observation concerning carriers of pathogenic germline mutations at codon 634 and the polymorphic variant L769L. Apparently, the age at onset of the disease in carriers of the germline mutation and the polymorphic variant was lower than in carriers of only the RET codon 634 pathogen mutation, Fig. 1.

In our material, similarly as Baumgartner-Parzer et al. (12), we found that individuals harbouring a RET codon 791 mutation always harboured the L769L polymorphism as well. As the most recent data $(6,24)$ suggested, mutation F791Y may be much more frequent than it had previously been thought to occur.

In the studied material, unlike in the case of the polymorphic change $\mathrm{L} 769 \mathrm{~L}$, the analysis of the frequency of the polymorphic variant S836S occurring in exon 14 of the RET gene showed no statistically significant differences in the frequency of heterozygous polymorphisms between patients with sporadic MTC and the control group. Our results match the findings of other authors $(12,13 ; 22,25)$ who found no significant differences in the frequency of these changes between patients with sporadic MTC and non-affected individuals.

However, many authors $(26,27,28)$ claim that the molecular variant S836S in the RET gene plays a more important role in the pathogenesis of MTC. Griseri et al. (29), 
suggested that the polymorphic variant S836S could increase RET receptor TK activity (similarly to the germline gain-of-function mutation). In the authors' opinion, the polymorphic change S836S could lead to incorrect protein synthesis or amino acid substitution in the protein caused by similarity and erroneous recognition of aminoacyl-tRNA.

Robledo et al. (30) noticed that G691S/S904S haplotype of RET may influence the age at onset in MEN2A patients. The change G691S is mainly responsible for that effect, however it cannot be ruled out that polymorphic change S904S can lead to production of different amounts of mRNA. The frequency of the polymorphic change S904S in our study population is similar in the study and control groups. The occurrence of the polymorphic change itself is, probably, not a factor predisposing to MTC development. It is possible, however, that the occurrence of that polymorphic variant, accompanying other more serious changes, can accelerate and modify the oncogenic effect induced by those changes.

The mechanisms by which polymorphic changes in the RET gene affect synthesis of the protein encoded by this gene are still not properly understood. It is presumed that their activity results in interrupted transcript splicing. As early as in the late 90s, Borrego et al. (26) put forward a thesis that polymorphic variants may cause new, alternative splicing sites (acceptor, donor or enhancer) to appear, which lead to the synthesis of incomplete proteins or erroneous ligand binding, microRNA binding, change of structure and mRNA stability as well as a number of copies and also the change in the structure of proteins caused by interference (slowing down) of translation $(31,32) \_O u r$ analyses based on bioinformatic methods made possible to draw several conclusions concerning mRNA conservation in the case of presence of various polymorphic variants in the RET gene.

Codon usage bias refers to differences among organisms in the frequency of occurrence of synonymous codons in mRNA. Optimal codons reflect the composition of their respective genomic tRNA pool. It is thought that using optimal codons help to achieve faster translation rates and higher accuracy. Folding of the beta sheet occurs slower than the alpha helix formation (33). If translation rate is changing before the process of beta sheet formation is finished, newly synthesized sequence influence the structure earlier (or later) then usual 
and may effect on the folding of the protein. If the mutation is a change of optimal to less frequent codon, it can lead to translation or protein folding disorders, because of ribosome stalling (pause). SNP S836S and S904S are changes to less frequent codons, so ribosome stalling can happen. In case of SNP L679L, where the codon with higher codon usage appear, the sheet may not finish creating the structure, when helix appears (34), Tab.5. As a consequence, there is not enough space to create the appropriate structure. This can lead to changes in kinase activity and/or specificity and, as a result, influence disease sympthomes. We may thus assume, on the basis of the studies conducted, that the molecular polymorphic variants in the RET gene play a very important role in the predisposition to sporadic medullary thyroid carcinoma and modify the age at onset of this disease.

Declaration of interest: The authors report no conflicts of interest. The authors alone are responsible for the contents and writing of the paper. 


\section{REFERENCES}

1. Takahashi M, Ritz J, Cooper GM. Activation of the novel human transforming gene RET by DNA rearrangement. Cell 42: 581-588, 1985.

2. Takahashi M, Buma $\mathrm{Y}$, Iwamoto $\mathrm{T}$, Inaguma $\mathrm{Y}$, Ikeda $\mathrm{H}$, Hiai H. Cloning and expression of the ret proto-oncogene encoding a receptor tyrosine kinase with two potential transmembrane domains. Oncogene 3 (5): 571-578, 1988.

3. Manie S, Santoro M, Fusco A, Billaud M. The ret receptor: function in development and dysfunction in congenital malformation. Trends Genet 17 (10): 580-589, 2001.

4. Kodama Y, Asai N, Kawai K, Jijiwa M, Murakumo Y, Ichihara M. The RET protooncogene: A molecular therapeutic target in thyroid cancer. Cancer Sci 96 (3): 143-148, 2005.

5. de Groot JWB, Links TP, Plukker JTM, Lips CJM, Hofstra RMW. RET as a diagnostic and therapeutic target in sporadic and hereditary endocrine tumors. Endocr Rev 27 (5): 535-560, 2006.

6. Paszko Z, Sromek M, Czetwertyńska M, Skasko E, Czapczak D, Wisniewska A, et al. The occurrance and the type of germline mutations in the RET gene in patients with medullary thyroid carcinoma and their unaffected kindred's from Central Poland. Cancer Invest 25 (8): 742-9, 2007.

7. Arighi E, Borrello MG, Sariola M. RET tyrosine kinase signaling in development and cancer. Cytokine Growth Factor Rev 16: 441-467, 2005.

8. Ponder BAJ. The phenotypes associated with ret mutations in the multiple endocrine neoplasia type 2 syndrome. Cancer Res (suppl.) 59: 1736-1742, 1999.

9. Hansford JR, Mulligan LM. Multiple endocrine neoplasia type 2 and RET from neoplasia to neurogenesis. J Med Genet 37: 817-827, 2000.

10. Human Genome Projekt Information: September 19, 2008. Available at: http://www.ornl.gov/sci/techresources/Human_Genome/faq/snps.shtml 
11. Gartner W, Mineva I, Daneva T, Baumgartner-Parzer S, Niederle B, Vierhapper $\mathrm{H}$, et al. A newly identified RET proto-oncogene polymorphism is found in a high number of endocrine tumor patients. Hum Genet 117: 143-153, 2005.

12. Baumgartner-Parzer SM, Lang R, Wagner L, Heinze G, Niederle K, Kaserer K, et al. Polymorphisms in exon 13 and intron 14 of the RET proto-oncogene: Genetic modifiers of Medullary Thyroid Carcinoma? J Clin Endocrinol Metab 90 (11): 6232-6236, 2005.

13. Elisei R, Cosci B, Romei C, Bottici V, Sculli M, Lari R, et al. RET exon 11 (G691S) Polymorphism is significantly more frequent in sporadic medullary thyroid carcinoma than in the general population. J Clin Endocrinol \& Metab 89 (7): 35793584, 2004.

14. Hofacker IL, Fontana W, Stadle PF, Bonhoeffer LS, Tacker M, Schuster P. Fast Folding and Comparison of RNA Secondary Structures. Monatsh.Chem. 125: 167-188, 1994.

15. Darty K, Denise A, Ponty Y. VARNA: Interactive drawing and editing of the RNA secondary structure. Bioinformatics 25 (15): 1974-1975, 2009

16. Betts L, Spremulli LL. Analysis of the role of the Shine-Dalgarno sequence and mRNA secondary structure on the efficiency of translational initiation in the Euglena gracilis chloroplast atpH mRNA. J. Biol. Chem. 269: 26456-26463, 1994.

17. Berard I, Kraimps JL, Savagner F, Murat A, Renaudin K, Nicolli-Sire P, et al. Germline-sequence variants S836S and L769L in the REarranged during Transfection (RET) proto-oncogene are not associated with predisposition to sporadic medullary carcinoma in the French population. Clin Genet 65 (2): 150-2, 2004.

18. Costa P, Domingues R, Sobrinho LG, Bugalho MJ. RET polymorphisms and sporadic medullary thyroid carcinoma in Portuguese population. Endocrine 27 (3): 239-43, 2005. 
19. Fernandez RM, Navarro E, Antinolo G, Ruiz-Ferrer M, Borrego S. Evaluation of the role RET polymorphisms/haplotypes as modifier loci for MEN2, and analysis of the correlation with the type of RET mutation in a series of Spanish patients. Int J Mol Med 17(4): 575-81, 2006.

20. Gursoy A, Erdogan MF, Erdogan G. Significance of the RET proto-oncogene polymorphisms in Turkish sporadic medullary thyroid carcinoma patients. J Endocrinol Invest 29, 2006.

21. Wohllk GN, Soto CE, Bravo AM, Becker P. G691S, L769L and S836S ret protooncogene polymorphisms are not associated with higher risk to sporadic medullary thyroid carcinoma in Chilean patients. Rev Med Chil 133(4): 397-402, 2005.

22. Wiench M, Włoch J, Wygoda Z, Gubala E, Oczko M, Pawlaczek A, et al. RET polymorphisms in codons 769 and 836 are not associated with predisposition to medullary thyroid carcinoma. Cancer Detect Prev 28: 231-236, 2004.

23. Weinhaeusel A, Scheuba C, Lauss M, Kriegner A, Kaserer K, Vierlinger K, Haas OA, Niederle B. The influence of gender, age, and RET polymorphisms on C-cell hyperplasia and medullary thyroid carcinoma. Thyroid 18 (12): 1269-76, 2008

24. Vierhapper H, Rondot S, Schulze E, Wagner L, Hanslik S, Niederle B, et al. Primary hyperparathyroidism as the leading symptom in a patent with a Y791F RET mutation. Thyroid 15(11): 1303-8, 2005.

25. Patocs A, Valkusz Z, Igaz P, Balogh K, Toth M, Varga I, et al. Segregation of the V804L mutation and S836S polymorphisms of exon 14 of the RET gene in an extended kindred with familial medullary thyroid cancer. Clin Genet 63(3): 219-23, 2003.

26. Borrego S, Saez ME, Ruiz A, Balogh K, Toth M, Varga I, et al. Specific polymorphisms in the RET proto-oncogene are over-represented in patients with 
Hirschsprung disease and may represent loci modifying phenotypic expression. J Med Genet 36(10): 771-4, 1999.

27. Gimm O, Neuberg DS, Marsh DJ, Dahia PLM, Cuong Hoang-Vu, Raue F, et al. Over-representation of a germline RET sequence variant in patients with sporadic medullary thyroid carcinoma and somatic RET codon 918 mutation. Oncogene 18: 1369-1373, 1999.

28. Ruiz A, Antinolo G, Fernandez RM, Eng C, Marcos I, Borrego S. Germline sequence variant S836S in the RET proto-oncogene is associated with low level predisposition to sporadic medullary thyroid carcinoma in the Spanish population. Clin Endocrinol 55(3): 399-402, 2001.

29. Griseri P, Sancandi M, Patrone G, Bocciardi R, Hofstra R, Ravazzolo R, et al. A single-nucleotide polymorphic variant of the RET proto-oncogene is underrepresented in sporadic Hirschsprung disease. Eur J Hum Genet 8: 721$724,2000$.

30. Robledo M, Gil L, Pollan M, Cebrian A, Ruiz S, Azanedo M, Benitez J, Menarguez J, Rojas JM. Polymorphisms G691S/S904S of RET as Genetic Modyfiers of MEN2A. Cancer Research 63: 1814-1817, 2003.

31. Parmley JL, Hurt LD. How do synonymous mutations affect fitness? Bioassay 29(6): 515-9, 2007.

32. Kimchi-Sarfaty C, Oh JM, Kim IW, Sauna ZE, Calcagno AM, Ambudkar SV, et al. A „silent” polymorphisms in the MDR1 gene changes substrate specificity. Science 26: 525-8, 2007.

33. Muñoz V, Thompson PA, Hofrichter J, Eaton WA. Folding dynamics and mechanism of $\beta$-hairpin formation. Nature 390: 196-199, 1997.

34. Nakamura Y, Gojobori T, Ikemura T. Codon usage tabulated from the international DNA sequence databases. Nucleic Acids Research 26 (1): 334-334, 2007. 


\section{Legend}

Fig.1 Relationship between presence of SNP L769L and the age at onset of MTC symptoms in pathogenic 634 mutation carriers.

Fig.2 Minimal free energy (MFE) structures of mutated exon 13: A - wild type, B - pathogenic mutation, C - synonymous mutation ( SNP L769L, CUU $\rightarrow$ CUG), D - pathogenic mutation and synonymous mutation. 
Fig. 1

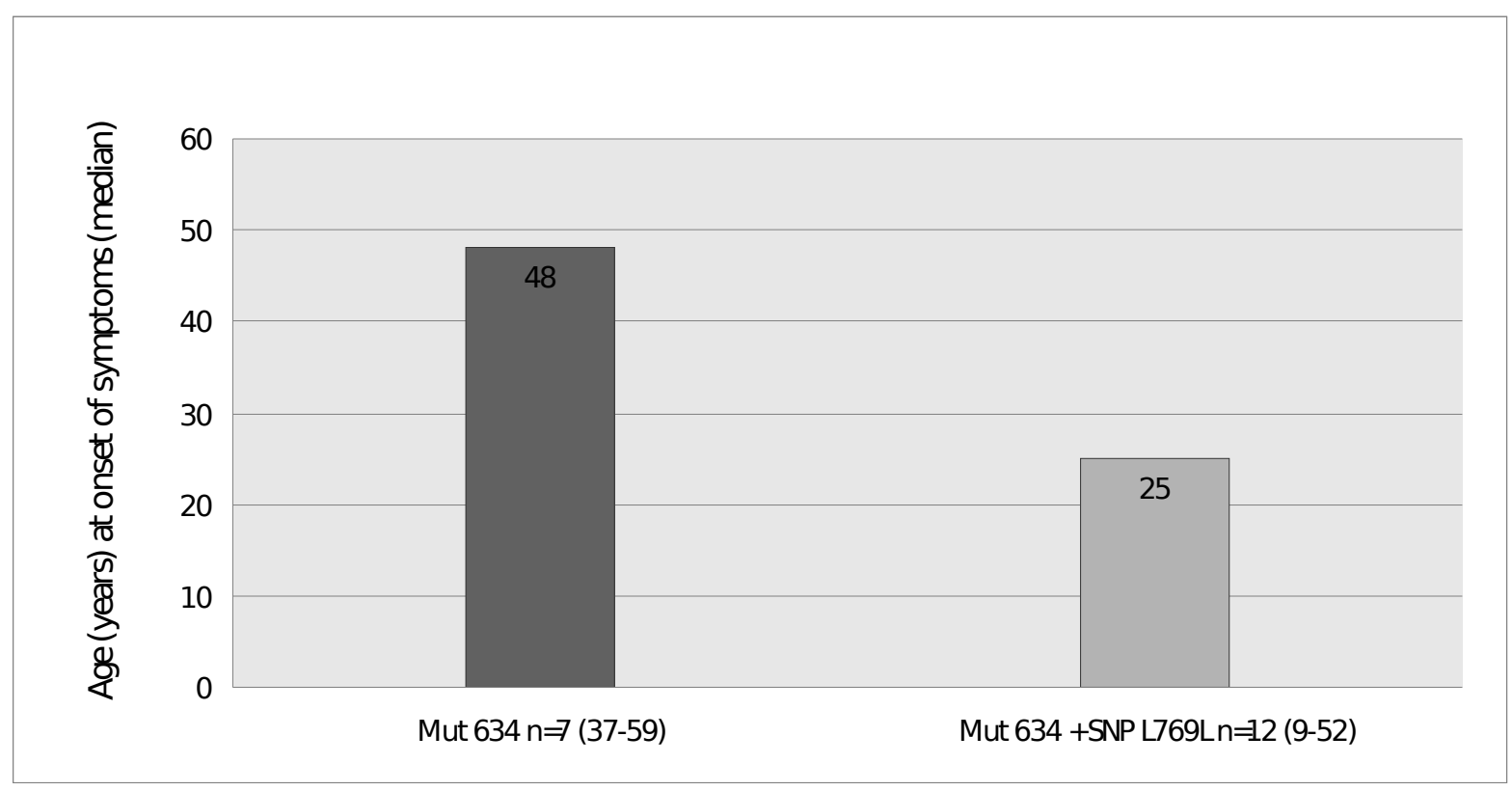


Fig. 2

A

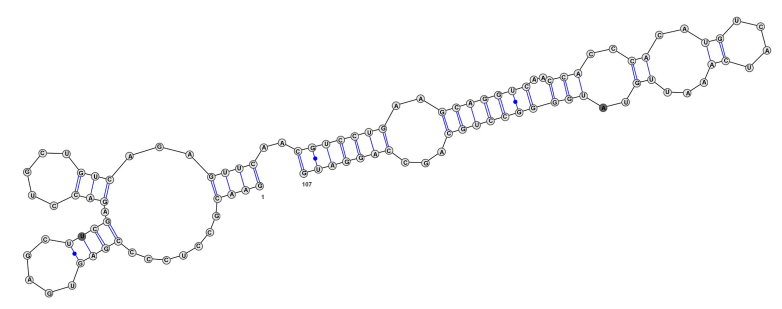

C

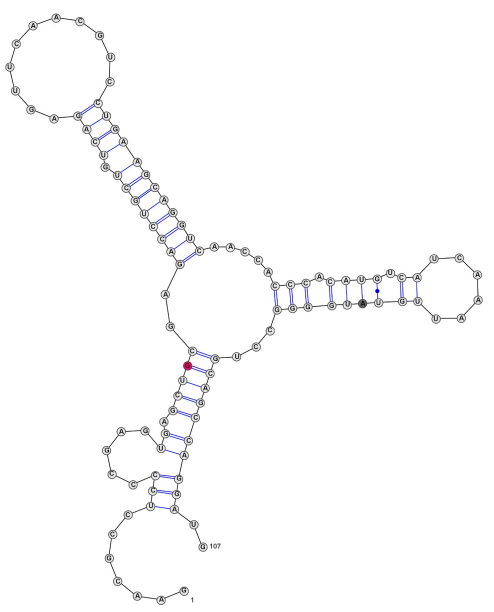

B

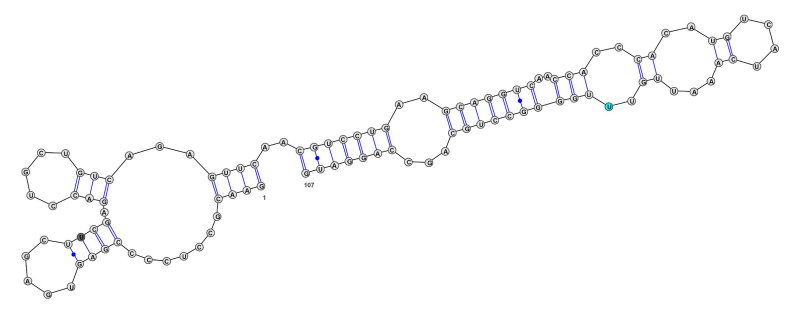

D

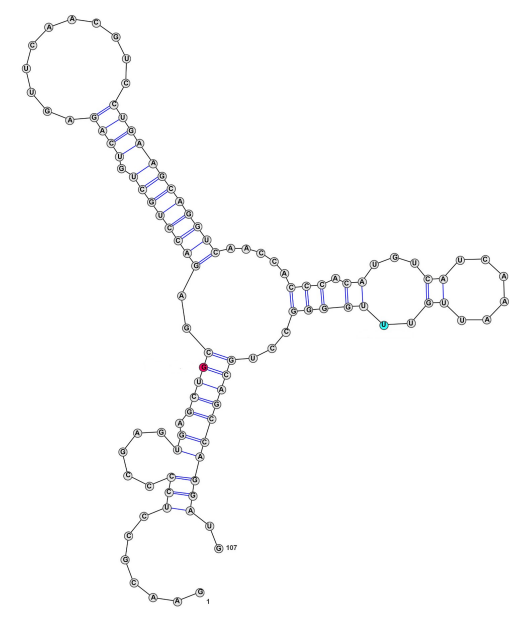


Tab.1 Allele frequencies of three exonic RET SNPs: L769L, S836S and S904S in sporadic MTC patients and controls.

(A) number of hetero- or homozygous subjects, (T) total number of sMTC patients, (N) total number of control individuals

\begin{tabular}{|c|c|c|c|c|}
\hline \multirow{2}{*}{ Allele } & sporadic MTC (\%) & control (\%) & \multirow{2}{*}{ OR $(95 \% \mathrm{Cl})$} & \multirow{2}{*}{ Statistic significance } \\
\hline & A / T (\%) & A / N (\%) & & \\
\hline \multicolumn{5}{|c|}{ SNP L769L } \\
\hline CTT/CTG & $105 / 217(48.3)$ & $166 / 420$ (39.5) & $1.43(1.03-1.99)$ & $p=0.0394^{*}$ \\
\hline CTG/CTG & 19/217 (8.7) & $36 / 420(8.5)$ & & $p=0.9374$ \\
\hline СTT/CTG + CTG/CTG & $124 / 217$ (57.14) & $202 / 420$ (48.09) & $1.43(1.03-2.00)$ & $p=0.0374^{\star}$ \\
\hline \multicolumn{5}{|c|}{ SNP S836S } \\
\hline AGCIAGT & $14 / 217(6.4)$ & 28/411 (6.8) & & $p=0.8633$ \\
\hline AGT/AGT & $0 / 217(0.0)$ & 2/411 (0.4) & & $p=0.7760$ \\
\hline AGCIAGT + AGT/AGT & $14 / 217(6.45)$ & $30 / 411(7.3)$ & & $p=0.6923$ \\
\hline \multicolumn{5}{|c|}{ SNP S904S } \\
\hline TCC/TCG & $66 / 217(30.4)$ & $135 / 420(32.14)$ & & $p=0.7227$ \\
\hline TCG/TCG & $8 / 217(3.6)$ & $8 / 420(1.9)$ & & $p=0.2735$ \\
\hline TCC/TCG + TCG/TCG & 74/217 (34.1) & $143 / 420(34.0)$ & & $p=0.9892$ \\
\hline
\end{tabular}


Tab 2. Age at onset of symptoms for SMTC (median) in patients with different sequence variants in codon 769 RET gene in whom no RET pathogenic mutation was found.

Median of age (years) at onset of SMTC symptoms

Statistical significance

\begin{tabular}{cccccc}
\hline CTT/CTT $n=89$ & CTT/CTG $n=103$ & CTG/CTG $n=18$ & \multicolumn{3}{c}{ Alleles compared } \\
A & B & C & A/B & A/C & B/C \\
\hline $52(15-85)$ & \multirow{2}{*}{$55(18-83)$} & $40.5(22-70)$ & $p=0.63$ & $p=0.021^{*}$ & $p=0.015$
\end{tabular}

The research covered a group of 210 patients for whom age at onset of symptoms of sporadic MTC could be established

Wild type(A), heterozygous change (B), homozygous change (C). All patients were non-carriers of pathogenic mutations in gene RET. 
Tab.3 The frequencies of RET variants 769/CTG, 836/ATG and 904/CTG in carriers of pathogenic mutations 791 and 634.

Number of hetero-and homozygous carriers of SNP / Total number of mutation carriers (\%)

\begin{tabular}{lcc}
\hline & Mutation: F791Y & Mutations: C634R, C634S, C634G \\
SNP: L769L & $20 / 20(100)$ & $13 / 22(59,0)$ \\
SNP: S836S & $0 / 20(0)$ & $2 / 23(8.6)$ \\
SNP S904S & $3 / 14(21,4)$ & $7 / 15(46,6)$ \\
\hline
\end{tabular}


Tab. 4 Energy parameters of predicted RNA structures for selcted exons without (WT - wild type) and with mutations: exon 13 with pathogenic mutation Y791F (TAT $\rightarrow$ TTT) and/or synonymous mutation SNP L769L (CUU $\rightarrow$ CUG); exon 14 with synonymous mutation SNP S836S (AGC $\rightarrow$ AGU); exon 15 with synonymous mutation SNP S904S $(\mathrm{UCC} \rightarrow$ UCG); exons 11-13 with pathogenic mutation C634R $($ TGC $\rightarrow$ CGC) and/or synonymous mutation SNP L769L (CUU $\rightarrow$ CUG).

Minimum free energy, ensemble diversity and the free energy of the thermodynamic ensemble are predicted by RNAfold software as described in materials and methods.

\begin{tabular}{|c|c|c|c|c|c|c|c|c|c|c|c|c|}
\hline & \multicolumn{4}{|c|}{ Exon 13} & \multicolumn{2}{|c|}{ Exon 14} & \multicolumn{2}{|c|}{ Exon 15} & \multicolumn{4}{|c|}{ Exon 11-13 } \\
\hline & $\begin{array}{l}\text { WT } \\
\text { (Fig. 1A) }\end{array}$ & $\begin{array}{l}\text { Mut } 791 \\
\text { (Fig. 1C) }\end{array}$ & $\begin{array}{c}\text { Mut 791 + } \\
\text { L769L } \\
\text { (Fig. 1B) }\end{array}$ & $\begin{array}{l}\text { L769L } \\
\text { (Fig. 1D) }\end{array}$ & WT & S836S & WT & S904S & WT & Mut C634R & L769L & $\begin{array}{c}\text { Mut C634R } \\
+ \text { L769L }\end{array}$ \\
\hline $\begin{array}{c}\text { Minimum free } \\
\text { energy (MFE) } \\
{[\mathrm{kcal} / \mathrm{mol}]}\end{array}$ & $-31,9$ & $-32,7$ & -35 & $-37,4$ & $-94,1$ & -93 & $-42,7$ & $-42,4$ & $-196,9$ & $-198,9$ & $-203,5$ & $-205,5$ \\
\hline $\begin{array}{l}\text { The ensemble } \\
\text { diversity }\end{array}$ & 38,98 & 36,41 & 17 & 9,11 & 63,82 & 55,61 & 23,08 & 18,38 & 96,38 & 94,59 & 86,09 & 84,27 \\
\hline $\begin{array}{l}\text { The free energy } \\
\text { of the } \\
\text { thermodynamic } \\
\text { ensemble } \\
\text { [kcal } / \mathrm{mol}]\end{array}$ & $-35,53$ & $-35,49$ & $-37,91$ & $-39,63$ & $-97,63$ & $-97,41$ & $-45,94$ & $-45,42$ & $-213,92$ & $-220,39$ & $-215,57$ & $-186,4$ \\
\hline
\end{tabular}


Tab. 5 Codon Usage comparison. Values of codon usage (frequency per thousand) in Homo sapiens were taken from Codon Usage Database [36].

\begin{tabular}{ccc}
\hline SNP & Codon change & Codon Usage (Hom Sapiens) \\
\hline L769L & CUU $\rightarrow$ CUG & $13,2 \rightarrow 39,6$ \\
S836S & AGC $\rightarrow$ AGU & $19,5 \rightarrow 12,1$ \\
S904S & UCC $\rightarrow$ UCG & $17,7 \rightarrow 4,4$ \\
\hline
\end{tabular}

